Somnologie $2020 \cdot 24: 3-4$

https://doi.org/10.1007/s11818-020-00247-8

Online publiziert: 26. März 2020

(c) Springer Medizin Verlag $\mathrm{GmbH}$, ein Teil von Springer Nature 2020

\title{
Wolfgang Galetke
}

Klinik für Pneumologie, VAMED Klinik Hagen Ambrock, Hagen, Deutschland

\section{Pneumologische Krankheitsbilder und Schlaf}

Veränderungen der Atmung und der Lungenfunktion lassen sich auch beim Gesunden im Schlaf beobachten. Mit dem Übergang vom Wachsein in den Non-REM-Schlaf kommt es zu einer zunehmenden Abnahme des Tidalvolumens, welches im REM-Schlaf den niedrigsten Wert erreicht. Die Atemfrequenz bleibt dagegen im Non-REMSchlaf weitgehend unverändert, sodass es zu einem Abfall des Atemminutenvolumens auf etwa $85 \%$ des Wertes im Wachzustand kommt. Im REMSchlaf dominiert ein eher unregelmäßiges Atemmuster mit Schwankungen der Atemfrequenz und der Amplitude des Atemflusses. Durch diese Veränderungen kann es beim Gesunden durch den Abfall des Atemminutenvolumens im Schlaf zu einem Anstieg des Kohlendioxidpartialdrucks und zu einem Abfall des Sauerstoffpartialdrucks kommen.

Die genannten physiologischen Veränderungen der Atmung und der Lungenfunktion im Schlaf interagieren auf verschiedene Weise mit den pathophysiologischen Aspekten der Atmung im Schlaf bei den chronisch-obstruktiven Atemwegserkrankungen, nämlich der chronisch-obstruktiven Lungenerkrankung (COPD) und dem Asthma bronchiale. Nächtliche Beschwerden sind bei Patienten mit einem Asthma bronchiale durch zirkadiane Schwankungen von Cortisol und Adrenalin, eine in der Nacht verstärke systemische und lokale Inflammation der Atemwege und eine verstärke unspezifische bronchiale Hyperreagibilität hervorgerufen. Weitere Aspekte sind der Anstieg des Atemwegswiderstandes in der Nacht sowie möglicherweise nächtliche Refluxepisoden. Bei der COPD spielt dagegen eher eine im Schlaf zunehmende Hypo- ventilation bei fortgeschrittenen Stadien der Erkrankung eine bedeutsame Rolle. Infolge der Lungenüberblähung weist das Diaphragma bereits im Wachzustand einen reduzierten Wirkungsgrad auf. Dies kann durch den Einsatz der Atemhilfsmuskulatur noch kompensiert werden. Fällt diese Kompensation durch einen zunehmenden Tonusverlust der Atemmuskulatur im Schlaf bei COPDPatienten weg, kann es zu einer relevanten Hypoventilation kommen. Diese tritt zunächst im REM-Schlaf auf, kann aber bei fortgeschrittener COPD in allen Schlafphasen mit besonderer Betonung der REM-Phasen beobachtet werden. Beide Erkrankungen, die COPD und das Asthma bronchiale, interagieren im Schlaf auch mit der Funktion der oberen Atemwege, sodass die Kombination aus diesen chronisch-obstruktiven Atemwegserkrankungen und der obstruktiven Schlafapnoe gehäuft auftritt. Für die Kombination aus OSA und COPD wurde der Begriff „Overlap-Syndrom “ geprägt. Das Overlap-Syndrom geht mit einer schlechteren Prognose einher als eine chronisch-obstruktive Lungenerkrankung ohne zusätzliche schlafbezogene Atmungsstörungen. Daher ist es von großer Bedeutung, auch bei diesen pneumologischen Erkrankungen an das zusätzliche Vorhandensein von nächtlichen Atmungsstörungen zu denken, diese $\mathrm{zu}$ diagnostizieren und $\mathrm{zu}$ behandeln.

Die idiopathische Lungenfibrose (IPF) ist eine progredient verlaufende interstitielle Lungenerkrankung mit schlechter Prognose. Schlafstörungen und schlafbezogene Atmungsstörungen bei der IPF sind insofern von Bedeutung, als es Hinweise für eine Mortalitätssteigerung und damit Prognoseverschlech- 
terung bei Patienten mit ausgeprägten nächtlichen Hypoxämien und zusätzlich bestehender pulmonaler Hypertonie gibt. Das Fehlen einer effektiven kausalen Therapiemöglichkeit bei der IPF macht die Optimierung der Schlafund Lebensqualität $\mathrm{zu}$ einem wichtigen Behandlungsziel.

Für Patienten, die auf einer Intensivstation behandelt werden, steht die schwere, zum Intensivaufenthalt führende Grunderkrankung sicherlich im Vordergrund. Darüber hinaus zeigen aber wissenschaftliche Studien einen signifikanten Zusammenhang zwischen einem gestörten Schlaf auf der Intensivstation und einem verlängerten Intensivaufenthalt sowie einer erhöhten Mortalität auf. Gründe für die Schlafstörungen auf der Intensivstation sind vielfältig und hän- gen eher nicht mit der Grunderkrankung zusammen, sondern werden unter anderem durch pflegerische und ärztliche Tätigkeiten, Lärmbelästigung, ungünstige Lichtverhältnisse etc. hervorgerufen. Die Erfassung der Schlafqualität intensivmedizinisch versorgter Patienten steht häufig nicht im Fokus der behandelnden Ärzte. Gerade die Entwicklung eines Delirs auf einer Intensivstation kann aber durch eine Verbesserung der Schlafqualität der Patienten verhindert werden. Dies hat unmittelbar Einfluss auf die Liegedauer auf der Intensivstation und auf die Sterblichkeit im Krankenhaus. Die dargestellten Zusammenhänge stellen nur einige Aspekte deskomplexen Zusammenhangs zwischen Schlafstörungen und pulmonalen Erkrankungen dar, die in dieser Ausgabe der Somnologie beleuchtet werden.
Die Lektüre der Artikel soll auch dazu beitragen, dem Schlaf bei der Diagnose und Behandlung pneumologischer Erkrankungen eine noch größere Aufmerksamkeit zukommen zu lassen.

\section{Korrespondenzadresse}

Prof. Dr. med. Wolfgang Galetke

Klinik für Pneumologie, VAMED Klinik Hagen

Ambrock

Ambrocker Weg 60, 58091 Hagen, Deutschland wolfgang.galetke@vamed-gesundheit.de

Interessenkonflikt. W. Galetke gibt an, dass kein Interessenkonflikt besteht.

\section{e.Medpedia: Die neue Online-Enzyklopädie für Ärzte}

e.Medpedia ist die neue digitale Enzyklopädie für Ärzte und ermöglicht das schnelle Nachschlagen auf Basis medizinischer Standardwerke von Springer. Die über Peer-ReviewVerfahren begutachteten Einträge werden von über 2.800 erfahrenen klinischen Experten verfasst und fortlaufend aktualisiert.

- e.Medpedia enthält alle Inhalte von über 20 etablierten Referenzwerken von Springer

- Inklusive unzähliger Abbildungen, klinischer Bilder, Tabellen und Schemata sowie Videos

- Verfasst von über 2.800 renommierten Fachärzten, gesichert durch Peer Review-Verfahren

- Komfortable Suchfunktion mit schneller Erkennung der Suchwörter

- Über 7.000 Querverlinkungen zwischen den einzelnen Einträgen

- Die bestehenden Einträge werden fortlaufend aktualisiert

- Weitere Fachgebiete werden kontinuierlich erweitert

- Mobile Nutzung über Smartphones - online und offline mit der e.Medpedia App für iOS- und Android-Geräte

Weitere Informationen:

www.springermedizin.de/emedpedia

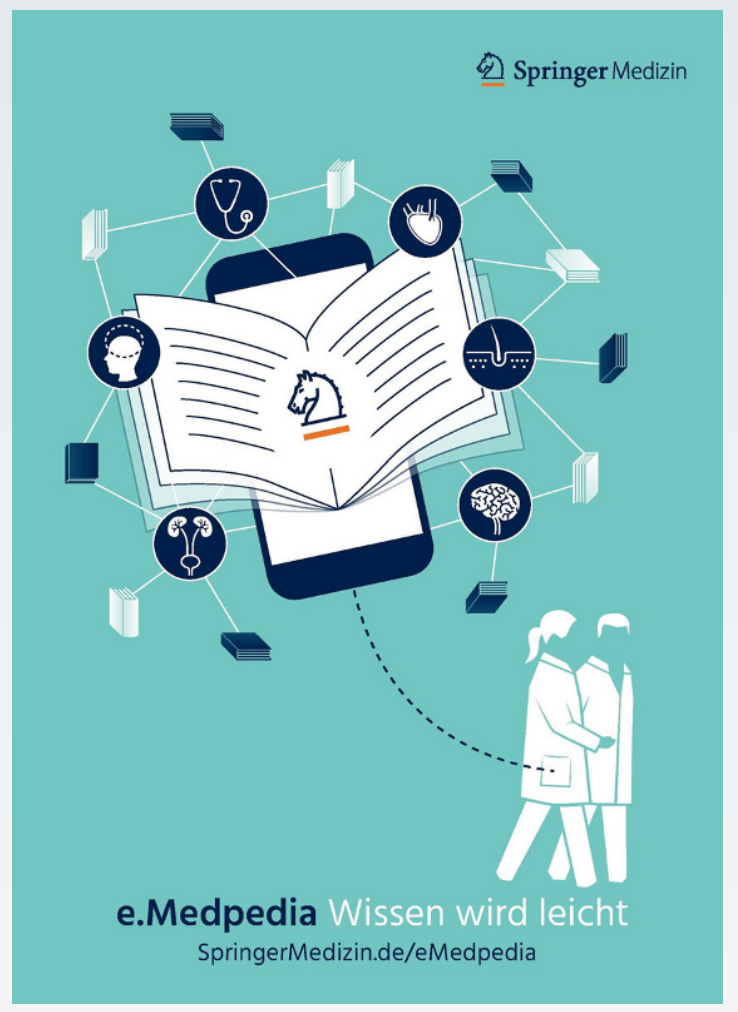

